\title{
Revista Colombiana de

\section{Diagnóstico automático del síndrome coronario agudo utilizando un sistema multiagente basado en redes neuronales}

\author{
John Jaime Sprockel Díaz ${ }^{a, *}$, Juan José Diaztagle Fernández ${ }^{\mathrm{a}, \mathrm{b}}$ \\ y Enrique González Guerrero ${ }^{c}$ \\ a Medicina Interna, Fundación Universitaria de Ciencias de la Salud-Hospital de San José, Bogotá, Colombia \\ b Departamento de Ciencias Fisiológicas, Universidad Nacional de Colombia, Bogotá, Colombia \\ c Departamento de Ingeniería de Sistemas y Computación, Pontificia Universidad Javeriana, Bogotá, Colombia
}

Recibido el 29 de abril de 2016; aceptado el 21 de noviembre de 2016

Disponible en Internet el 28 de abril de 2017

\section{PALABRAS CLAVE \\ Diagnóstico; \\ Síndrome coronario \\ agudo; \\ Infarto agudo de \\ miocardio; \\ Angina inestable; \\ Dolor torácico}

\begin{abstract}
Resumen
Introducción: Por tratarse de una tarea altamente compleja y de importancia clínica, el diagnóstico del síndrome coronario agudo se presta para su exploración por medio de modelado mediante sistemas inteligentes.

Objetivo: desarrollar un sistema multiagente que ensamble las decisiones de varias redes neuronales para el diagnóstico del dolor torácico enfocado a los síndromes coronarios agudos. Metodología: estudio de pruebas diagnósticas en el que se entrenan un conjunto de redes neuronales con una precisión cercana al 70\%, que luego son ensambladas mediante tres sistemas de votación para luego adicionar el resultado de redes especiales en poblaciones particulares y seleccionar la mejor configuración que hará parte de un sistema multiagente para el diagnóstico del dolor torácico.

Resultados: Se generaron 84 redes con precisión promedio del $72 \%$ en pruebas; al ensamblarse aumentan dicha precisión hasta llegar a un máximo del $84 \%$ que tras la adición de los grupos especiales alcanza el $89 \%$. Se escoge una conformación que brinda una sensibilidad del $96 \%$ con una especificidad del $77 \%$, con valores predictivos positivo y negativo de 87 y $93 \%$ respectivamente para el diagnóstico de síndrome coronario agudo.
\end{abstract}

\footnotetext{
* Autor para correspondencia.

Correo electrónico: jjsprockel@fucsalud.edu.co (J.J. Sprockel Díaz).
} 
Conclusiones: Es posible desarrollar una herramienta para el diagnóstico automático del síndrome coronario agudo a partir de un sistema multiagente que ensamble la disposición tomada por un conjunto de redes neuronales artificiales, cuyo rendimiento permite su consideración para su implementación dentro de un sistema de soporte a las decisiones clínicas.

(c) 2016 Sociedad Colombiana de Cardiología y Cirugía Cardiovascular. Publicado por Elsevier España, S.L.U. Este es un artículo Open Access bajo la licencia CC BY-NC-ND (http:// creativecommons.org/licenses/by-nc-nd/4.0/).

\author{
KEYWORDS \\ Diagnosis; \\ Acute coronary \\ syndrome; \\ Acute myocardial \\ infarction; \\ Unstable angina; \\ Chest pain
}

\section{Automatic diagnosis of acute coronary syndrome using a multi-agent system based} in neural networks

\begin{abstract}
Introduction: Because it is a highly complex task of a great clinical importance, the diagnosis of acute coronary syndromes allows for their analysis by means of intelligent system models. Motivation: To develop a multi-agent system that assembles the decisions of several neural networks for the diagnosis of chest pain with a focus on acute coronary syndromes.

Methods: A study of diagnostic tests where a series of neural networks are trained with a precision close to $70 \%$, and are later on assembled with three voting systems. Then the results of special networks on specific populations are added to select the best configuration that will make part of a multi-agent system for diagnosing chest pain.

Results: A total of 84 networks were generated, with an average precision of $72 \%$ during testing; once assembled this precision rises up to a maximum of $84 \%$, which then reaches $89 \%$ when the special groups are included. A configuration that offers a sensitivity of $96 \%$ with a specificity of $77 \%$ and positive and negative predictive values of 87 and $93 \%$ respectively is chosen for the diagnosis of acute coronary syndrome.

Conclusions: It is possible to develop a tool for the automatic diagnosis of acute coronary syndrome using a multi-agent system that assembles the dispositions taken by a set of artificial neural networks. Its performance allows taking it into consideration for implementing it within a clinical decision-making support system.

(C) 2016 Sociedad Colombiana de Cardiología y Cirugía Cardiovascular. Published by Elsevier España, S.L.U. This is an open access article under the CC BY-NC-ND license (http:// creativecommons.org/licenses/by-nc-nd/4.0/).
\end{abstract}

\section{Introducción}

El diagnóstico del síndrome coronario agudo representa una tarea compleja, implica la puesta en consideración de las características demográficas, la presentación clínica y los antecedentes obtenidos a través de la historia clínica, junto con los resultados del electrocardiograma y los marcadores de lesión miocárdica confirmados luego por alguna estrategia de estratificación coronaria invasiva o no. Su principal forma de presentación, el dolor torácico, implica tener en cuenta un gran número de condiciones que aunque en su mayoría sean benignas, diluyen otras que ponen en riesgo la vida (como la embolia pulmonar y la disección aórtica entre otras).

Desde el campo de la inteligencia artificial una herramienta muy usada son las redes neuronales artificiales, las cuales pueden describirse como paradigmas computacionales basados en modelos matemáticos con capacidad de un fuerte patrón de reconocimiento. Consisten en un algoritmo de cálculo que se basa en una analogía del sistema nervioso ${ }^{1}$. Representan el campo de los sistemas inteligentes que ha sido evaluado de manera más extensa y completa dentro del área del diagnóstico del síndrome coronario agudo. Desde los primeros trabajos a principios de los años 90 por
Baxt $^{2}$ y Furlong ${ }^{3}$, se ha comprobado su alta efectividad para la discriminación del infarto ${ }^{4} \mathrm{u}$ otros síndromes coronarios agudos $^{5}$; con datos procedentes del electrocardiograma ${ }^{6}$ o de éste más la historia clínica y marcadores de daño miocardico ${ }^{7}$, se ha realizado además la validación cruzada en sitios diferentes al que fueron desarrolladas ${ }^{8}$ y manejo en el contexto de series de tiempo ${ }^{9}$. En todos estos casos se han logrado obtener niveles de precisión cercanos y superiores al $90 \%$.

Los sistemas multiagentes son una colección de agentes independientes que se comunican con el fin de cooperar en la solución conjunta de una tarea compleja ${ }^{10}$. Se entiende por agente una entidad de software que de forma autónoma maneja diversas entradas desde su ambiente con el que interactúa, y que persigue de manera proactiva el logro de sus objetivos cooperando o compitiendo con otros agentes. Una de las formas en que un grupo de agentes que persiguen un objetivo común en el reconocimiento de un patrón puede interactuar para reunir sus resultados son los diversos sistemas de ensamble, entre los que se encuentran: votación, promedio bayesiano, bagging, boosting, modelos basados en árboles, mezcla condicionada (mezclas de modelos de regresión lineal, logísticos y mezclas de expertos) ${ }^{11}$. La combinación de ambas estrategias podría llegar a brindar un 
panorama prometedor para modelar, mediante un sistema informático, el proceso de diagnóstico.

En este trabajo se presenta el proceso mediante el cual se llevaron a cabo varios experimentos para el desarrollo de un sistema multiagente basado en redes neuronales y sistemas de ensamble para el apoyo al diagnóstico del dolor torácico enfocado en el síndrome coronario agudo.

\section{Metodología}

Se llevó a cabo un estudio de pruebas diagnósticas. En una fase inicial se entrenó y probó un conjunto de RNA para el diagnóstico de síndrome coronario agudo que luego se integró bajo diferentes formas de ensamble. Finalmente, se seleccionó la mejor combinación para su aplicación dentro de un sistema multiagente.

Las diferentes RNA fueron entrenadas a partir de 307 pacientes procedentes de dos bases de datos: una constituida por 159 pacientes mayores de 18 años que consultaron por dolor torácico como motivo de consulta principal al servicio de urgencias, en el período comprendido entre el 20 febrero y el 30 de octubre de 2012; esta cohorte fue derivada de un estudio institucional en el cual se evaluó la implementación de una ruta crítica en el síndrome coronario agudo en el Hospital San José de Bogotá12 . La segunda base de datos estuvo constituida por 148 pacientes, recogidos de manera prospectiva, mayores de 18 años, hospitalizados, con sospecha clínica de síndrome coronario agudo entre el 25 de julio de 2013 y el 1 de agosto de 2014 diseñada específicamente para el presente trabajo; incluyó además pacientes del Hospital Universitario San José Infantil de Bogotá. Ambos trabajos fueron autorizados por el comité de investigaciones y ética de la Fundación Universitaria de Ciencias de la Salud y el Hospital San José.

El estándar de referencia para el diagnóstico de infarto se basó en la tercera redefinición de infarto ${ }^{13}$ y para el caso de la angina inestable en la presencia de datos clínicos compatibles (principalmente dolor torácico de reciente comienzo, in crescendo o en reposo) junto con la positividad en alguna estrategia de estratificación coronaria invasiva o no invasiva.

Las variables de entrada para la discriminación del dolor torácico se fundamentaron en los datos que constituyen las escalas de Braunwald ${ }^{14}$, Florencia $^{15}$ y Sanchis $^{16}$, que han sido desarrolladas para el estudio y clasificación de riesgo de los dolores torácicos bajo sospecha de un síndrome coronario agudo. Todas tienen en consideración variables demográficas, características del dolor, antecedentes y resultados del electrocardiograma y marcadores de lesión miocárdica.

Las redes neuronales fueron entrenadas y probadas en la población general o en los diversos subgrupos de población, aplicando un algoritmo genético para la selección de los pesos de las redes. Se dividió la población en un $70 \%$ para entrenamiento y un $30 \%$ para pruebas (17). Con el fin de detectar las mejores formas de ensamble de las redes neuronales se realizó un experimento factorial $3^{3}$ dentro de un modelo de efectos fijos, no replicado, con un nivel de significancia del $5 \%$. Se tomó como variable de respuesta la precisión diagnóstica de cada uno de las arquitecturas de ensamble, probadas en el $30 \%$ del conjunto total de datos (92 ejemplos).
Los factores estudiados fueron ${ }^{17}$ :

- Número de datos que se tuvieron en cuenta para el entrenamiento y pruebas de las redes.

- Número de redes escogidas a partir de cada uno de los conjuntos de datos de entrenamiento (escalas de Braunwald, Florencia y Sanchis).

- Heurística empleada dentro del sistema de ensamble de las decisiones:

- Votación simple

- Votación ponderada por valores predictivos

- Votación ponderada por razón de verosimilitud (likelihood ratios, LR).

En un siguiente paso se realizó una corrida con los mismos tratamientos descritos incluyendo dentro del ensamble el resultado de los agentes especializados en ciertos grupos de interés (diabéticos, mujeres, mayores de 75 años y con historia de enfermedad renal).

\section{Resultados}

En la tabla 1 se listan las características de los pacientes de las dos bases de datos tenidas en cuenta para los experimentos. A partir de las corridas del algoritmo genético realizado, se seleccionaron 84 RNA con un nivel de precisión que rondaba el $70 \%$, divididas de la siguiente manera: 72 para el diagnóstico de síndrome coronario agudo en toda la población (8 por cada uno de 3 niveles de número de datos de entrenamiento en los 3 datos de entrada procedentes de cada escala), 12 en subpoblaciones específicas (mujeres, mayores de 75 años, diabéticos y con antecedente de enfermedad coronaria.

En el análisis de las RNA individuales se encontró un promedio de precisión durante el desarrollo del $76,7 \%$ y del $72 \%$

Tabla 1 Características de los pacientes

\begin{tabular}{|c|c|c|}
\hline & $\begin{array}{l}\text { Primera base } \\
\text { de datos } \\
n=159\end{array}$ & $\begin{array}{l}\text { Segunda base } \\
\text { de datos } \\
n=148\end{array}$ \\
\hline Procedencia & HSJ, feb-oct 2012 & $\begin{array}{l}\text { HSJ y HUISJ, jul } \\
2013-A g o ~ 2014\end{array}$ \\
\hline $\begin{array}{l}\text { Diagnósticos, } n \\
(\%)\end{array}$ & $\begin{array}{l}\text { Anginas } 41(25,8 \%) \\
\text { Infartos } 37(23,3 \%) \\
\text { No coronario } 81 \\
(50,9 \%)\end{array}$ & $\begin{array}{l}\text { Anginas } 28(18,9 \%) \\
\text { Infartos } 68(46,0 \%) \\
\text { No coronario } 52 \\
(35,1 \%)\end{array}$ \\
\hline $\begin{array}{l}\text { Edad } \\
\text { (promedio) }\end{array}$ & 61,4 años & 64,1 años \\
\hline Mujeres, n (\%) & $72(45,3 \%)$ & 61 (41,2\%) \\
\hline $\begin{array}{l}\text { Estratificación } \\
\text { no invasiva, n } \\
\text { (\%) }\end{array}$ & $100(62,9 \%)$ & $126(85,1 \%)$ \\
\hline $\begin{array}{l}\text { Coronariografía, } \\
\text { n (\%) }\end{array}$ & $50(31,4 \%)$ & $99(66,9 \%)$ \\
\hline $\begin{array}{l}\text { Procedencia } \\
\text { de los datos }\end{array}$ & $\begin{array}{l}\text { Escala de } \\
\text { Braunwald }\end{array}$ & $\begin{array}{l}\text { Escala de } \\
\text { Braunwald, } \\
\text { Florencia y Sanchis }\end{array}$ \\
\hline
\end{tabular}

HSJ: Hospital San José, HISJ: Hospital Universitario Infantil de San José. 
Tabla 2 Resultados de los experimentos en las 27 combinaciones de ensamble con y sin la inclusión de los subgrupos (mujeres, diabéticos, mayores de 75 años e historia de enfermedad coronaria)

\begin{tabular}{|c|c|c|c|c|}
\hline Número de redes & $\begin{array}{l}\text { Nivel de datos de } \\
\text { entrenamiento }\end{array}$ & Tipo de ensamble & Precisión & $\begin{array}{l}\text { Precisión incluyendo } \\
\text { subgrupos }\end{array}$ \\
\hline 2 & Alto & Simple & 80 & 80 \\
\hline 5 & Alto & Simple & 80 & 83 \\
\hline 8 & Alto & Simple & 78 & 80 \\
\hline 2 & Medio & Simple & 78 & 80 \\
\hline 5 & Medio & Simple & 79 & 80 \\
\hline 8 & Medio & Simple & 79 & 81 \\
\hline 2 & Bajo & Simple & 72 & 76 \\
\hline 5 & Bajo & Simple & 80 & 79 \\
\hline 8 & Bajo & Simple & 79 & 82 \\
\hline 2 & Alto & Ponderada por VP & 82 & 86 \\
\hline 5 & Alto & Ponderada por VP & 82 & 88 \\
\hline 8 & Alto & Ponderada por VP & 83 & 88 \\
\hline 2 & Medio & Ponderada por VP & 82 & 83 \\
\hline 5 & Medio & Ponderada por VP & 85 & 85 \\
\hline 8 & Medio & Ponderada por VP & 82 & 86 \\
\hline 2 & Bajo & Ponderada por VP & 77 & 83 \\
\hline 5 & Bajo & Ponderada por VP & 84 & 84 \\
\hline 8 & Bajo & Ponderada por VP & 84 & 84 \\
\hline 2 & Alto & Ponderada por LR & 83 & 85 \\
\hline 5 & Alto & Ponderada por LR & 84 & 89 \\
\hline 8 & Alto & Ponderada por LR & 85 & 88 \\
\hline 2 & Medio & Ponderada por LR & 82 & 80 \\
\hline 5 & Medio & Ponderada por LR & 80 & 82 \\
\hline 8 & Medio & Ponderada por LR & 78 & 80 \\
\hline 2 & Bajo & Ponderada por LR & 76 & 78 \\
\hline 5 & Bajo & Ponderada por LR & 82 & 82 \\
\hline 8 & Bajo & Ponderada por LR & 83 & 84 \\
\hline
\end{tabular}

VP: valor predictivo, LR: likelihood ratio.

(con un rango que iba desde 60 a $81 \%$ ) en la fase de pruebas en los 92 ejemplos. En los resultados globales de desempeño de las diferentes combinaciones examinadas se encontró una variabilidad importante, obteniéndose en la mayoría de los casos altos niveles de sensibilidad (91-100\%) y más bajos de especificidad (36-67\%). Al centrar el análisis en el resultado de la precisión global de la prueba (tabla 2) se encontró que en la mayoría de los casos el rendimiento de los diferentes tratamientos supera al de los agentes individuales (rango entre 72 y $81 \%$ ); así, los sistemas ponderados tienen mejor desempeño que la votación simple.

El análisis de varianza (ANOVA) encuentra que los factores evaluados son significativos. En promedio, el sistema votación ponderada según valores predictivos fue mejor que los otros dos evaluados. Al realizar las pruebas adicionando las redes neuronales especializadas en subgrupos, se halló un incremento significativo de la precisión diagnóstica; los tratamientos con mejor desempeño fueron con las redes entrenadas con un nivel alto de ejemplos, con 5 y 8 agentes por cada escala usada y con la ponderación tanto por LR como por valores predictivos ( 89 y $88 \%$ ).

El resultado final (fig. 1) es un aplicativo en el que, luego del ingreso de los datos del paciente a través de la interface de usuario, se da inicio a un sistema multiagente constituido por un agente gestor general del proceso que posee la información global del diagnóstico del dolor torácico, un agente administrador de fase de diagnóstico de síndrome coronario agudo que crea los siete agentes que contienen las redes neuronales para el diagnóstico general: 3 que contienen cada una 5 redes neuronales entrenadas a partir de los datos de cada una de las escalas utilizadas (Braunwald, Florencia y Sanchis) y 4 agentes con las redes especializadas en subgrupos, 3 redes neuronales para mujeres, diabéticos y mayores de 75 años, y 2 redes neuronales para los que tenían antecedentes de enfermedad coronaria. Finalmente, se ensambla la decisión mediante una votación ponderada basada en razón de verosimilitud dentro del administrador de fase $^{17}$.

Esta conformación del sistema multiagente brinda una sensibilidad del $96 \%$ con una especificidad del $77 \%$, una precisión del $89 \%$, con valores predictivos positivo y negativo de 87 y $93 \%$ respectivamente para el diagnóstico de síndrome coronario agudo.

\section{Discusión}

En este informe se presenta una propuesta de un sistema desarrollado sobre un marco experimental robusto en el cual se integran las decisiones de un conjunto de RNA para el diagnóstico de síndrome coronario agudo usando un sistema de ensamble de decisiones al interior de un sistema 


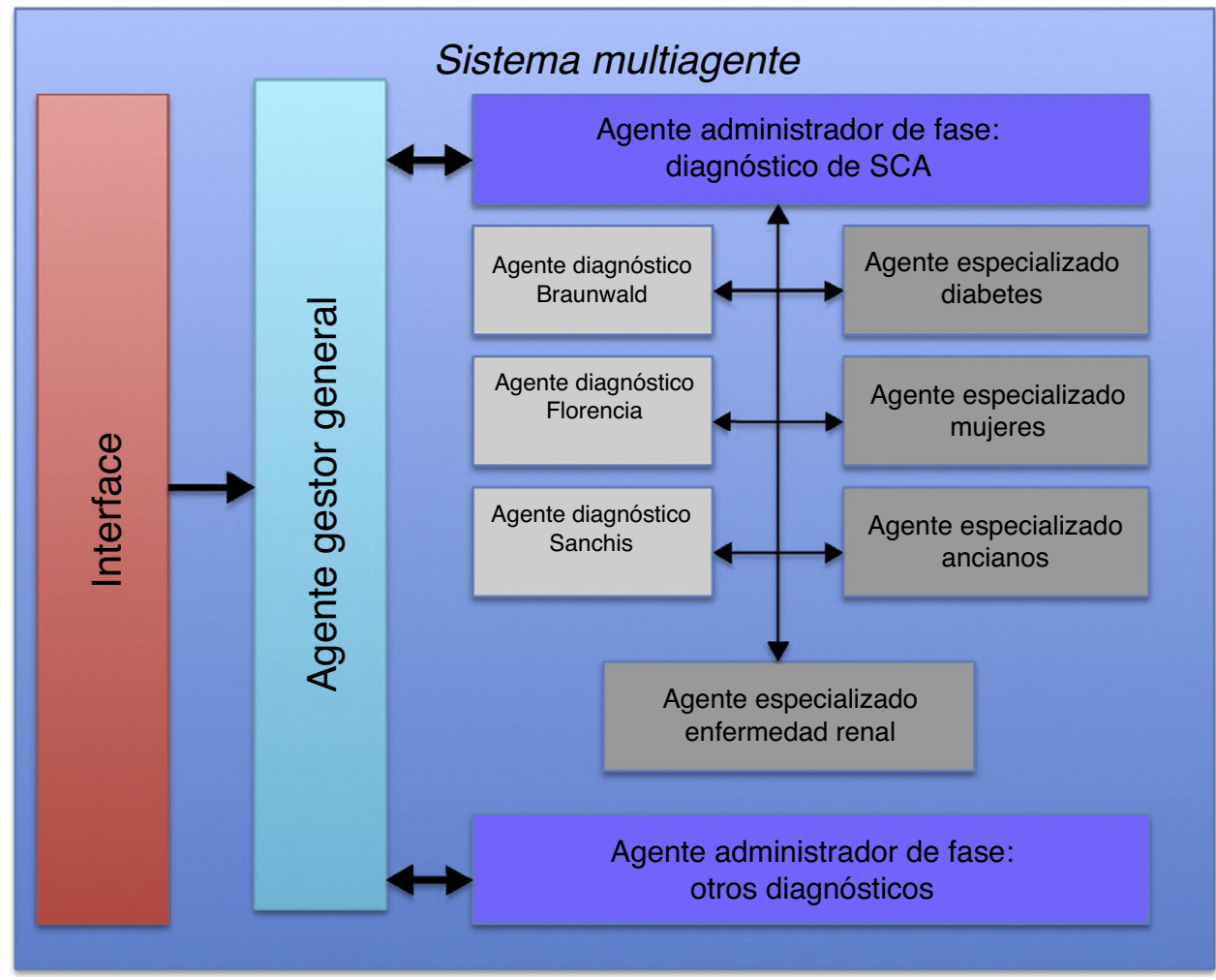

Figura 1 Organización del sistema multiagente para el diagnóstico de síndrome coronario agudo (ver descripción en el texto).

multiagente. Las RNA fueron intencionalmente diseñadas para tener un desempeño regular (alrededor del 70\%) con lo que se esperaba (como se logró) magnificar el efecto del ensamble.

El alto desempeño diagnóstico logrado en este experimento, está por encima de la mayor parte de los reportes de desempeño del personal médico, tanto general como especializado $2,18,19$, lo cual haría viable su uso como un sistema de soporte para las decisiones clínicas. Sin embargo, sus resultados deben ser corroborados en una fase posterior. Es así, que además de atractivo por lo innovador, representa oportunidades reales para la optimización del proceso de atención en una entidad causante del mayor número de muertes en el mundo y cuyo retraso en el diagnóstico se ve reflejado en un aumento de los eventos adversos.

En la bibliografía consultada se encontraron dos trabajos en los que se utilizaron los sistemas basados en ensamble de decisión de redes neuronales para el diagnóstico de síndrome coronario agudo dentro de un sistema multiagente. En el primero ${ }^{20}$, se utilizó bagging y cross-spliting para el análisis de los datos procedentes de 634 electrocardiogramas (130 con diagnóstico de síndrome coronario agudo); los resultados fueron muy similares a los del presente reporte, aunque con un menor rendimiento, área bajo la curva ROC (AUC) de 0,81; el rendimiento fue superior al de cada una de las redes neuronales que lo conformaba. Por otro lado, en un segundo estudio se desarrolló y probó un sistema multiagente en el cual se ensamblaron herramientas de sistemas inteligentes en el diagnóstico de la enfermedad coronaria, mediante varias técnicas de boosting: AdaBoost, MultiBoost y LogitBoost $^{21}$; la base de datos estuvo constituida por 270 pacientes con 13 variables en la Cleveland
Clinic Foundation, con 120 pacientes con enfermedad coronaria. Se obtuvieron altos niveles de desempeño, siendo la más alta la del AdaBoost (precisión 97,6\%, sensibilidad $97,6 \%$, especificidad $97,6 \%$ con un AUC 0,989).

Se reconoce como limitación el hecho que se trate apenas de un prototipo (en la actualidad se encuentra en curso un estudio para la validación cruzada del sistema). Por otro lado, no es claro si se mantiene el beneficio del ensamble cuando se usan redes neuronales con un mejor desempeño inicial (por encima del 90\%), como las encontradas en la revisión de la literatura.

Entre las fortalezas del estudio está el hecho de aprovechar aspectos del conocimiento médico en el problema diagnóstico del dolor torácico, utilizando los datos de las escalas aplicadas desde la clínica para la clasificación diagnóstica del síndrome coronario agudo mediante el uso de los datos de las escalas de Braunwald, Sanchis y Florencia; por otro lado, se desarrolló un gran número de redes neuronales procedentes de diferentes datos y poblaciones de entrenamiento, se contó con datos de dos centros hospitalarios al interior de la base de datos y se estructuró un marco experimental robusto analizando además varias formas de efectuar el ensamble.

Es evidente el cambio que ha venido gestándose en la medicina en los últimos lustros. Gracias a la conectividad y el amplio desarrollo de los sistemas de comunicación, ha sido posible la masificación de aplicativos que apoyan la actividad médica. En esa vía se hacen significativos los adelantos que permitan el apoyo del enfoque diagnóstico y de tratamiento de las patologías con mayor impacto para la salud. Desde este punto de vista, la población blanco está constituida principalmente por aquel médico de atención primaria 
o de urgencias con poca experiencia, recursos o apoyo institucional. El diagnóstico automático del síndrome coronario agudo se convierte en un enfoque atractivo para este tipo de desarrollo; los casos exitosos existen, aunque por múltiples razones no lo hemos visto reflejado en nuestra práctica diaria de la medicina.

\section{Conclusiones}

Es posible diseñar una herramienta para el diagnóstico automático del síndrome coronario agudo a partir de un sistema multiagente desarrollado para el diagnóstico del dolor torácico, que utiliza herramientas de ensamble de la decisión tomada por un conjunto de redes neuronales artificiales, y que además presenta un desempeño diagnóstico que supera el de cada una de las redes individualmente. La adición de redes especializadas en subgrupos, incrementa de manera considerable el rendimiento diagnóstico hasta poder ser considerado para su implementación en un sistema de soporte a las decisiones clínicas. Estos resultados deben tener una validación posterior para corroborar su utilidad.

\section{Responsabilidades éticas}

Protección de personas y animales. Los autores declaran que para esta investigación no se han realizado experimentos en seres humanos ni en animales.

Confidencialidad de los datos. Los autores declaran que han seguido los protocolos de su centro de trabajo sobre la publicación de datos de pacientes.

Derecho a la privacidad y consentimiento informado. Los autores declaran que en este artículo no aparecen datos de pacientes.

\section{Conflicto de intereses}

Los autores declaran no tener conflicto de intereses.

\section{Agradecimientos}

A los miembros del Departamento de Investigación y Estadística de la Fundación Universitaria Ciencias de la Salud-Hospital San José de Bogotá. Al personal médico y de enfermería del servicio de urgencias del Hospital San José de Bogotá.

\section{Bibliografía}

1. Sordo M, Vaidya S, Jain L. An introduction to computational intelligence in healthcare: new directions. Advanced computational intelligence paradigms in healthcare - 3. 3 rd. ed. Springer Berlin Heidelberg; 2008. p. 1-26.

2. Baxt WG. Use of an artificial neural network for the diagnosis of myocardial infarction. Ann Intern Med. 1991;115:843-8.

3. Furlong JW, Dupuy ME, Heinsimer JA. Neural network analysis of serial cardiac enzyme data. A clinical application of artificial machine intelligence. Am J Clin Pathol. 1991;96:134-41.
4. Ellenius J, Groth T, Lindahl B, Wallentin L. Early assessment of patients with suspected acute myocardial infarction by biochemical monitoring and neural network analysis. Clin Chem. 1997;43:1919-25.

5. Baxt WG, Shofer FS, Sites FD, Hollander JE. A neural computational aid to the diagnosis of acute myocardial infarction. Ann Emerg Med. 2002;39:366-73.

6. Al-Naima FM, Ali AH, Mahdi SS. Data acquisition for myocardial infarction classification based on wavelets and Neural Networks. IEEE; 2008 [cited 2013 Feb 9]. p. 1-6.

7. Bulgiba AM. Using neural networks and just nine patientreportable factors of screen for AMI. Health Informatics Journal. 2006;12:213-25.

8. Harrison RF, Kennedy RL. Artificial neural network models for prediction of acute coronary syndromes using clinical data from the time of presentation. Ann Emerg Med. 2005;46: 431-9.

9. Eggers KM, Ellenius J, Dellborg M, Groth T, Oldgren J, Swahn $E$, et al. Artificial neural network algorithms for early diagnosis of acute myocardial infarction and prediction of infarct size in chest pain patients. Intern J Cardiol. 2007;114:366-74.

10. Moreno A, Garbay C. Software agents in health care. Artif Intell Med. 2003;27:229-32.

11. Bishop C. Combining models. Pattern recognition and machine learning. Chapter 14: $1^{\text {st }}$. ed. Singapur: Springer-Verlag New York; 2006. p. 653-76.

12. Sprockel JJ, Diaztagle JJ, Chaves WG, Simon C, Hernandez JI. Estructuración e implementación de una ruta crítica para el manejo de pacientes con síndrome coronario agudo en el Servicio de Urgencias del Hospital San José de Bogotá. Rev Colom Cardiol. 2013;20:262-74.

13. Thygesen K, Alpert JS, Jaffe AS, Simoons ML, Chaitman BR, White HD, et al. Third universal definition of myocardial infarction. Circulation. 2012;126:2020-35.

14. Braunwald E, Jones RH, Mark DB, Brown J, Brown L, Cheitlin $M D$, et al. Diagnosing and managing unstable angina. Agency for Health Care Policy and Research. Circulation. 1994;90: 613-22.

15. Conti A, Vanni S, Taglia BD, Paladini B, Magazzini S, Grifoni $S$, et al. A new simple risk score in patients with acute chest pain without existing known coronary disease. Am J Emerg Med. 2010;28:135-42.

16. Sanchis J, Bodí V, Núñez J, Bertomeu-González V, Gómez C, Bosch MJ, et al. New risk score for patients with acute chest pain, non-ST-segment deviation, and normal troponin concentrations: a comparison with the TIMI risk score. J Am Coll Cardiol. 2005;46:443-9.

17. Sprockel J, Gonzalez E. Rational agents federation through neural networks assembly in the diagnosis of acute coronary syndromes. In: Computing Colombian Conference (10CCC), 2015 10th. IEEE; 2015. p. 357-63.

18. Hirshberg AJ, Guttman TG. Criteria-based expert system for cardiac ischemia evaluation in the emergency department. Acad Emerg Med. 1996;3:689-93.

19. Dhawan A, Wenzel B, George S, Gussak I, Bojovic B, Panescu D. Detection of acute myocardial infarction from serial ECGs using a multilayer support vector machine. Conf Proc IEEE Eng Med Biol Soc. 2012;2012:2704-7.

20. Green M, Björk J, Forberg J, Ekelund U, Edenbrandt L, Ohlsson M. Comparison between neural networks and multiple logistic regression to predict acute coronary syndrome in the emergency room. Artificial Intelligence in Medicine. 2006;38:305-18.

21. Mandal I, Sairam N. Accurate prediction of coronary artery disease using reliable diagnosis system. J Med Syst. 2012;36:3353-73. 